\title{
44518 - RADIOGRAPHIC EVALUATION OF CERVICAL SPINE MOVEMENT DURING FLEXIBLE VIDEOBRONCHOSCOPY \& LO-PRO GLIDESCOPE; A RANDOMISED CONTROLLED TRIAL
}

Disclosure: Other financial or material support - Lo_Pro Glidescope provided by the manufacturer.

\author{
Atul Prabhu, University Health Network, Toronto Western Hospital, Toronto, ON, \\ Canada; \\ Daniel Wong, Toronto Western Hospital; \\ Santanu Chakraborty, Toronto Western Hospital; \\ E Massicotte, Toronto Western Hospital; \\ G Tan, Toronto Western Hospital; \\ $R$ Cooper, Toronto General Hospital;
}

INTRODUCTION: It is essential to prevent or minimize cervical spine movement in patients with c-spine injury. Flexible bronchospcopy (FB) is widely regarded as the gold standard for intubating patients with airway instability. The Lo-Pro Glidescope (LP-G) is a new improved video laryngoscope and may be associated with less c-spine movement. We aimed to compare cspine movement using fluoroscopy during intubation with FB versus LP-G.

METHODS: After ethics board approval 28 consenting adults with no cervical spine abnormality were randomized to be intubated with either a FB or LP-G. Demographic data was collected. During intubation continuous video fluoroscopy of the cervical spine was performed. A neuroradiologist and a spine surgeon assessed c-spine movement independently. The primary endpoint was maximum angulations during the intubation sequence (Occ-C1, C1-2, C2-4, and C4-5). Power of the study was determined by considering a 1-degree change in angulations as significant. Mann Whitney $U$ test was used to compare maximum range of motion and Wilcoxin signed rank test for interobserver differences.

RESULTS: No significant differences between FB and the LP-G groups in demographic data. Intubation times were comparable between LP-G (31+ $2 \mathrm{sec})$ and FB $(31+3 \mathrm{secs})$.

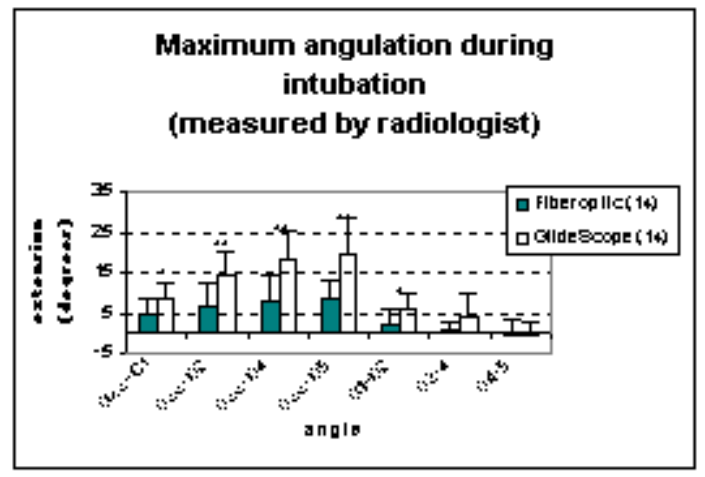


Max. change mean \&\#61617; SD, $* \mathrm{p}<0.05, * * \mathrm{p}<0.01$

DISCUSSION: The Lo-Pro Glidescope has significantly greater movement that the Flexible bronchoscopy.This study has shown that there is some movement associated even with the Flexible Bronchoscopy.

REFRENCES: 1.Turkstra TP. Crane RA. Pelz DM. Gelb AW. Anesth Analg. 2005:101:910-5

2. Cooper RM. Can J Anesth 2003;50:611-3. 